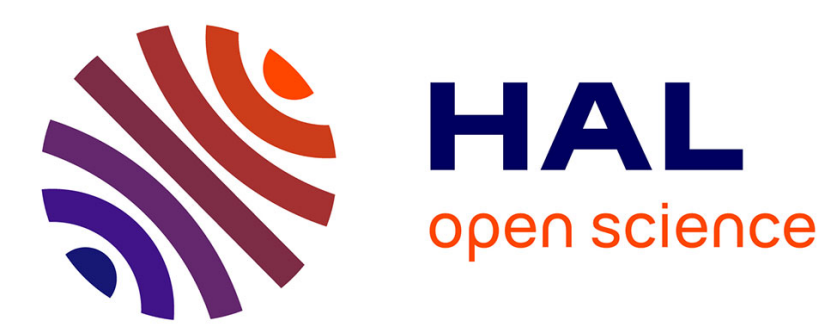

\title{
Micromechanical Studies of Cyclic Creep Fracture Under Stress-Controlled Loading
}

E. van Der Giessen, V. Tvergaard

\section{To cite this version:}

E. van Der Giessen, V. Tvergaard. Micromechanical Studies of Cyclic Creep Fracture Under Stress-Controlled Loading. Journal de Physique IV Proceedings, 1996, 06 (C6), pp.C6-449-C6-459. 10.1051/jp4:1996645 . jpa-00254478

\section{HAL Id: jpa-00254478 https://hal.science/jpa-00254478}

Submitted on 1 Jan 1996

HAL is a multi-disciplinary open access archive for the deposit and dissemination of scientific research documents, whether they are published or not. The documents may come from teaching and research institutions in France or abroad, or from public or private research centers.
L'archive ouverte pluridisciplinaire HAL, est destinée au dépôt et à la diffusion de documents scientifiques de niveau recherche, publiés ou non, émanant des établissements d'enseignement et de recherche français ou étrangers, des laboratoires publics ou privés. 


\title{
Micromechanical Studies of Cyclic Creep Fracture Under Stress-Controlled Loading
}

\author{
E. van der Giessen and V. Tvergaard* \\ Delft University of Technology, Laboratory for Engineering Mechanics, Mekelweg 2, 2628 CD Delft, \\ The Netherlands \\ * The Technical University of Denmark, Department of Solid Mechanics, Building 404, 2800 Lyngby, \\ Denmark
}

\begin{abstract}
This paper deals with a study of intergranular failure by creep cavitation under stress-controlled cyclic loading conditions. Loading is assumed to be slow enough that diffusion and creep mechanisms (including grain boundary sliding) dominate, leading to intergranular creep fracture. This study is based on numerical unit cell analyses for a planar polycrystal model with the grains and grain boundaries modeled individually, in order to investigate the interactions between the mechanisms involved and to account for the build-up of residual stress fields during cycling. The behaviour of a limiting case with a facet-size microcrack yields important insight for damage accumulation under balanced loading. Under unbalanced loading, the time-average accumulation of creep cavitation gives rise to macroscopic ratchetting, while its rate is demonstrated to depend subtly on material and loading conditions.
\end{abstract}

\section{INTRODUCTION}

For polycrystalline metals under monotonic loading at elevated temperatures, creep fracture occurs mainly by intergranular cavitation and microcracking (e.g. [1]). Cavities nucleate and grow most rapidly on grain boundary facets normal to the maximum principal tensile stress, and facet microcracks form by coalescence of cavities. The growth of individual cavities under such conditions due to grain boundary diffusion and creep has been studied in quite some detail (e.g. [2]), and using these results the development of cavitation damage and microcracking in polycrystalline aggregates has been investigated (e.g. $[3,4]$ ). These and several other studies have clearly demonstrated the interaction and competition between the various mechanisms involved under typical creep conditions with monotonic loading.

Under cyclic loading at elevated temperatures, both creep damage and fatigue are likely failure mechanisms (see e.g. [5]). In a range of very slow cycling there will be sufficient time for creep and diffusion mechanisms to dominate, leading to intergranular creep fracture, whereas a typical transgranular fatigue fracture tends to develop in the opposite situation of very rapid cycling. In the whole range between these two extremes, the failure process is usually termed creep-fatigue interaction (e.g. [6]).

For the range of slow cycling, where creep failure mechanisms dominate, there are a number of basic phenomena that are not well understood. One of the standing issues, for instance, is why some materials show cavitation even under balanced cyclic loading with zero mean stress. Diffusive cavity growth follows an equation linear in stress, which should not result in cavity growth under balanced cycling. Various explanations have been attempted to resolve this, but only with limited success.

The plane strain multi-grain cell model of Van der Giessen and Tvergaard $[4,7]$ has been used in [8] to study mechanisms of creep failure under cyclic loading, with focus on materials subject to a strain-controlled loading programme, as is often used in cyclic testing. The cell model represents a number of individual grains that deform by power-law creep, and it also accounts 


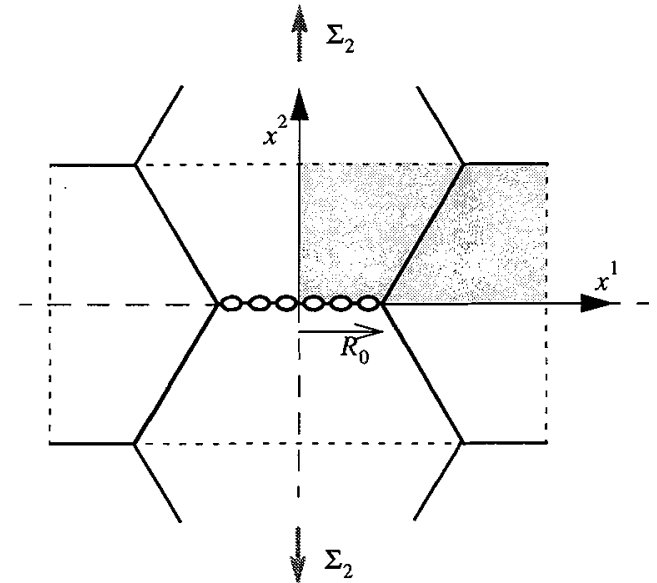

(a)

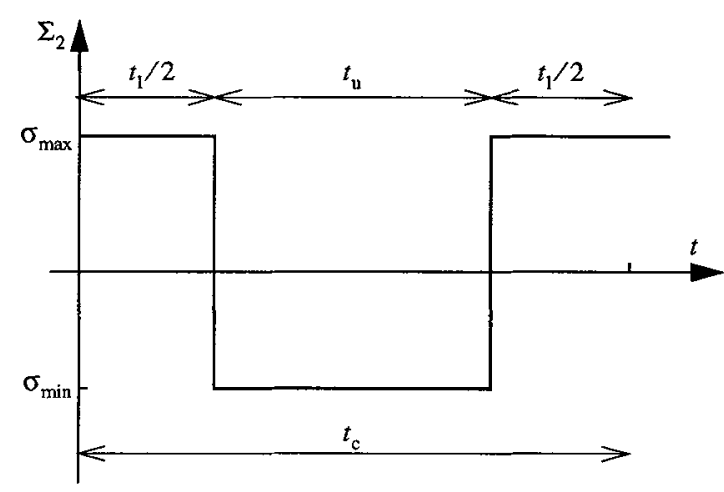

(b)

Figure 1: (a) Unit cell of the planar polycrystal material model, comprising two hexagonal grains. Only the shaded area is analyzed in view of symmetry. (b) Cyclic loading conditions in terms of the uniaxial macroscopic stress $\Sigma_{2}$.

for viscous grain boundary sliding, continuous nucleation of grain boundary cavities and cavity growth by coupled diffusion and creep; but fatigue failure mechanisms are not incorporated in the model. Thus, the results are only relevant to a range of relatively slow cycling, where intergranular creep failure mechanisms are completely dominant. The important additional dimension that the multi-grain model adds to the cyclic creep considerations in e.g. [5] is that it includes the internal stress re-distributions and corresponding residual stresses inside the grain aggregate in the course of the failure process. The studies in [8] have shown that rather important understanding of some features of cyclic creep damage behaviour is obtained by considering a facet-size microcrack. But, the studies accounting for cavity nucleation and growth, show a rather complex phenomenology in general, due to the build-up of residual stresses during cycling.

In the present paper the plane strain multi-grain cell model is subjected to stress-controlled cyclic loading, including cases of unbalanced cycling. Although unbalanced cycling was also considered in [8], e.g. in the form of a longer hold period in tension, this was done for equal strain amplitudes in tension and compression, thus eliminating any chance of ratchetting. But stresscontrolled cyclic loading gives a more realistic representation of the loading conditions in some structural systems, where ratchetting is an important failure phenomenon. This cyclic growth of the overall material straining is studied here together with the corresponding development of grain boundary cavitation.

\section{PROBLEM FORMULATION}

The model problem we use here to investigate cyclic creep is based on a special case of the multigrain model developed previously by the authors $[4,7]$. We refer to those references for details on the formulation and method of solution; here we shall only give a brief recapitulation. As a model for a planar polycrystalline material, we consider an aggregate of regular hexagonal grains with facet width $2 R_{0}$, oriented such that one of the three families of parallel facets is normal to the principal stress direction $x^{2}$, as shown in Fig. 1(a). Within this aggregate, we assume a unit cell comprising a number of grains; the present study will be limited to a small cell consisting only of two grains. 


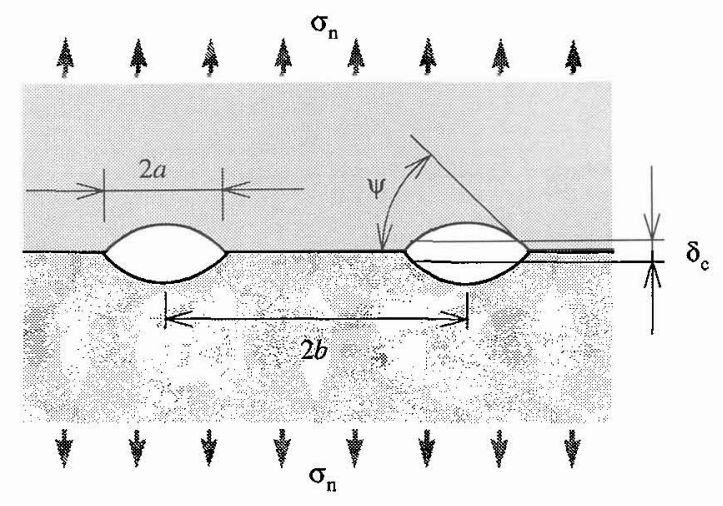

Figure 2: Geometry of spherical-caps shaped cavities on a grain boundary subjected to a remote facet normal stress $\sigma_{\mathbf{n}}$.

The material is taken to be subjected macroscopically to uniaxial (i.e. $\Sigma_{1}=0$ ) cyclic loading in the $x^{2}$ direction under plane strain conditions in the $x^{3}$ direction. Here, cyclic loading is stresscontrolled with the macroscopic stress $\Sigma_{2}$ being prescribed by a cyclic wave consisting of a period $t_{1}$ of constant maximal tensile stress $\sigma_{\max }$ and a period $t_{\mathrm{u}}$ of constant minimal stress $\sigma_{\min }$. Balanced loading corresponds to $t_{1}=t_{\mathrm{u}}$ and $\sigma_{\min } / \sigma_{\max }=-1$, and unbalance can be introduced either by letting $t_{\mathrm{u}} \neq t_{1}$ and/or $\sigma_{\min } / \sigma_{\max } \neq-1$. The cycle starts with a half period under $\sigma_{\max }$ in order to retain similarity with the strain-controlled loading in [8] which started at zero strain.

The following micromechanisms, typical for polycrystailine metals and alloys at elevated temperatures, are being accounted for in the model: (i) dislocation creep inside the grains, (ii) grain boundary sliding, and (iii) the nucleation and (iv) growth of cavitities on the grain boundaries.

For the description of the high-temperature response of the grains, we take the grain material to be isotropic for simplicity. Creep deformations of the grains are taken to be characterized by the power law relationship

$$
\dot{\epsilon}_{\mathrm{e}}^{\mathrm{C}}=\dot{\epsilon}_{0}\left(\frac{\sigma_{\mathrm{e}}}{\sigma_{0}}\right)^{n},
$$

between the effective Mises stress $\sigma_{\mathrm{e}}$ and the corresponding creep strain-rate $\dot{\epsilon}_{\mathrm{e}}^{\mathrm{C}}$ with a creep exponent $n$. The quanities $\dot{\epsilon}_{0}$ and $\sigma_{0}$ are reference strain-rate and stress quantities. At elevated temperatures, grains can slide relative to each other with a linearly viscous resistance against sliding. For given creep parameters in (1) and a given sliding viscosity, the relative contributions to the overall deformation depend on the stress level. For sufficiently low stress levels, grain boundary sliding takes place at negligible shear stresses (i.e. free sliding), whereas at large stresses, creep is so fast that there is insufficient time for sliding to take place. Here, we assume that conditions are such that sliding can be considered to be shear stress free.

Grain boundary cavitation takes place preferentially on grain boundary facets transverse to the maximal principal stress. In this explorative study, we confine attention to cases where only the central grain facet in the unit cell of Fig. 1(a) is prone to cavitation. Furthermore, we shall assume that all cavitities have either nucleated prior to the cyclic loading programme to be investigated, or nucleate only in the very first stages of cyclic loading over a time frame much smaller than the period of the cycle. Hence, we neglect any further cavity nucleation during cycling. The growth of cavities is governed by the following set of expressions that have been developed [3] on the basis of detailed investigations of the growth of individual cavities in, for example, $[2,9,10]$.

The size of a cavity (see Fig. 2), $a$, increases due to both grain boundary diffusion and creep of the grain material. If it is assumed that the tip angle $\psi$ maintains a constant value throughout 
the process, we can write $\dot{a}=\dot{V} /\left[4 \pi a^{2} h(\psi)\right]$ with $h(\psi)=\left[(1+\cos \psi)^{-1}-\frac{1}{2} \cos \psi\right] / \sin \psi$ the cavity shape parameter. The volumetric growth rate $\dot{V}$ is expressed as

$$
\dot{V}=\dot{V}_{1}+\dot{V}_{2}, \quad \text { for } a / L \leq 10, \quad f=\max \left[\left(\frac{a}{b}\right)^{2},\left(\frac{a}{a+1.5 L}\right)^{2}\right]
$$

where

$$
\begin{gathered}
\dot{V}_{1}=4 \pi \mathcal{D} \frac{\sigma_{\mathrm{n}}-(1-f) \sigma_{\mathrm{s}}}{\ln \left(\frac{1}{f}\right)-\frac{1}{2}(3-f)(1-f)}, \\
\dot{V}_{2}= \begin{cases}2 \pi \dot{\epsilon}_{\mathrm{e}}^{\mathrm{C}} a^{3} h(\psi) m\left[\alpha_{n}\left|\frac{\sigma_{\mathrm{m}}}{\sigma_{\mathrm{e}}}\right|+\beta_{n}(m)\right]^{n}, & \left|\frac{\sigma_{\mathrm{m}}}{\sigma_{\mathrm{e}}}\right|>1 \\
2 \pi \dot{\epsilon}_{\mathrm{e}}^{\mathrm{C}} a^{3} h(\psi)\left[\alpha_{n}+\beta_{n}(m)\right]^{n} \frac{\sigma_{\mathrm{m}}}{\sigma_{\mathrm{e}}}, & \left|\frac{\sigma_{\mathrm{m}}}{\sigma_{\mathrm{e}}}\right| \leq 1 .\end{cases}
\end{gathered}
$$

with

$$
m=\operatorname{sign}\left(\frac{\sigma_{\mathrm{m}}}{\sigma_{\mathrm{e}}}\right) .
$$

The parameter $\mathcal{D}=D_{\mathrm{B}} \delta_{\mathrm{B}} \Omega / k T$ in the growth rate expression (3) due to diffusion is the grain boundary diffusion parameter, with $D_{\mathrm{B}} \delta_{\mathrm{B}}$ denoting the boundary diffusivity, $\Omega$ the atomic volume, $k$ Boltzmann's constant and $T$ the absolute temperature. The sintering stress $\sigma_{s}$ in (3) is neglected relative to the facet normal stress $\sigma_{\mathrm{n}}$. Furthermore, in the creep growth expression (4), $\sigma_{\mathrm{m}}$ and $\sigma_{\mathrm{e}}$ are the average mean and Mises stress, respectively, remote from the void. The constants appearing in (4) are given by $\alpha_{n}=3 / 2 n$ and

$$
\beta_{n}(m)=\frac{(n-1)[n+g(m)]}{n^{2}} ; \quad g(1)=0.4319, \quad g(-1)=0.4031 .
$$

The small difference between $\beta_{n}(1)$ and $\beta_{n}(-1)$ [9] is accounted for, but we have not found any significant numerical influence in our computations. The length scale [2]

$$
L=\left[\mathcal{D} \sigma_{\mathrm{e}} / \dot{\epsilon}_{\mathrm{e}}^{\mathrm{C}}\right]^{1 / 3}
$$

is a key parameter governing the coupling between diffusive and creep contributions to void growth by way of the selection of the value for $f$ to be used in the diffusive growth expression (3). For large values of this length scale $L$, say $a / L<0.1$, cavity growth is dominated by diffusion, while for larger values of $a / L$ creep growth becomes more and more important. As a consequence, cavity growth in situations where $a / L<0.1$ or so, is likely to be constrained by creep of the material surrounding the cavitating facet, whereas this creep constraint reduces with increasing $a / L$ (see e.g. $[11,3])$.

The nucleation and growth of cavitities at some average half-spacing $b$ give rise to an average separation $\delta_{c}$ between the two adjacent grains (see Fig. 2). With nucleation being neglected here, the rate of change of this separation,

$$
\dot{\delta}_{\mathrm{c}}=\frac{\dot{V}}{\pi b^{2}}-\frac{2 V}{\pi b^{2}} \frac{\dot{b}}{b}
$$

is governed largely by the volumetric growth $\dot{V}$ of cavities according to (2). In addition, there is a relatively minor contribution of finite lateral straining in the plane of the grain boundary, expressed through $\dot{b} / b$. 
In cyclic creep, cavities may shrink to very small size under a compressive facet normal stress $\sigma_{\mathrm{n}}$ [cf. (3)]. However, we will assume here that the cavity nucleus still remains present, so that existing cavities do not completely disappear by sintering and therefore need not be re-nucleated under tension. When the ratio $a / b$ reaches the value 0.7 during the process, cavity coalescence is assumed to occur by failure of the ligament between cavities. Thus, a new microcrack is formed or an existing microcrack is extended. Under cyclic loading it may be possible that such a microcrack closes up again, but it is unlikely that previously coalesced cavities can heal to re-form cavities. Therefore, the microcracks are regarded to simply make contact again when the actual separation between grains has become as small as the separation $\delta_{\mathrm{c}}$ at coalescence, and the microcrack can immediately open-up again when loading becomes tensile.

The governing equations for the grains as well as the grain boundary layers accounting for cavitation and grain boundary sliding are formulated within a linear incremental framework, using a forward gradient integration scheme for numerical stability. Finite strains are accounted for. The solution is based on a standard finite element discretization of the grains and a special-purpose discretization of the grain boundaries. Here, a total of 206 quadrilateral elements are used withtin the quarter cell (see Fig. 1(a)) with 14 elements along half of the central facet.

\section{RESULTS}

The material parameters in this study are mostly chosen in conformity with the previous study [8] under strain-control. The grain material is taken to be specified by $\nu=0.3$ and $n=5$. The stress range during cycling is always specified so that the maximum tensile stress $\sigma_{\max }$ corresponds to $\Sigma_{\mathrm{e}} / E=0.5 \times 10^{-3}$ ( $E$ and $\nu$ are the Young's modulus and Poisson's ratio, respectively). Here, $\Sigma_{\mathrm{e}}$ is defined as the effective macroscopic Mises stress corresponding to pure plane strain creep under uniaxial stress $\Sigma_{2}$, i.e. $\Sigma_{\mathrm{e}}=\frac{1}{2} \sqrt{3}\left|\Sigma_{2}\right|$. With the macroscopic creep-rate parameter $\dot{E}_{\mathrm{e}}^{\mathrm{C}}$ defined through (1), this introduces the creep reference time $t_{\mathrm{R}}=\Sigma_{\mathrm{e}} /\left(E \dot{E}_{\mathrm{e}}^{\mathrm{C}}\right)$. The balanced cycling cases that will serve as references cases in this study are for $t_{1}=t_{\mathrm{u}}=0.1 t_{\mathrm{R}}$. The macroscopic strain response, $E_{2}$, during cyclic stressing is normalized by the strain range $\Delta \epsilon^{\mathrm{C}}=\dot{E}_{2} t_{1}$ that would be found under plane strain creep of a homogeneous material under the specified tensile stress.

\subsection{Behaviour of an open microcrack}

For the mechanisms considered in this study, creep failure involves three distinct time scales, namely that of creep flow, grain boundary diffusion and the loading. Under constant stress creep loading $\left(\sigma_{\min }=\sigma_{\max }\right)$, there is an important regime of stress and material parameters where diffusion is much faster than creep of the grains (i.e. $a / L$ according to (5) small [2]), leading to (diffusive) cavity growth being constrained by creep of the grain material [11]. In that regime, substantial stress re-distribution in the course of the process has reduced the normal stresses on cavitating facets far below the applied stress level. Therefore, the limiting problem of the behaviour of an open microcrack along a grain facet has been exploited to formulate models for constrained cavitation (see e.g. [3]). Under strain-controlled cyclic creep conditions, the behaviour of a microcrack along the central facet in Fig. 1 has also been helpful in understanding cyclic creep failure [8].

In Fig. 3 we therefore consider the corresponding case under balanced stress-controlled cycling (with the stress level and frequency as mentioned above). Because of free sliding along the inclined grain boundaries, the microcrack opens and closes uniformly. After the first half tensile wave, the opened-up microcrack (opening $\delta$ ) is completely closed quickly after loading changed to compression. The compressive creep rate is much lower than when the crack opened up. At the start of the next tensile wave, the microcrack opens up immediately again, so that crack closure 


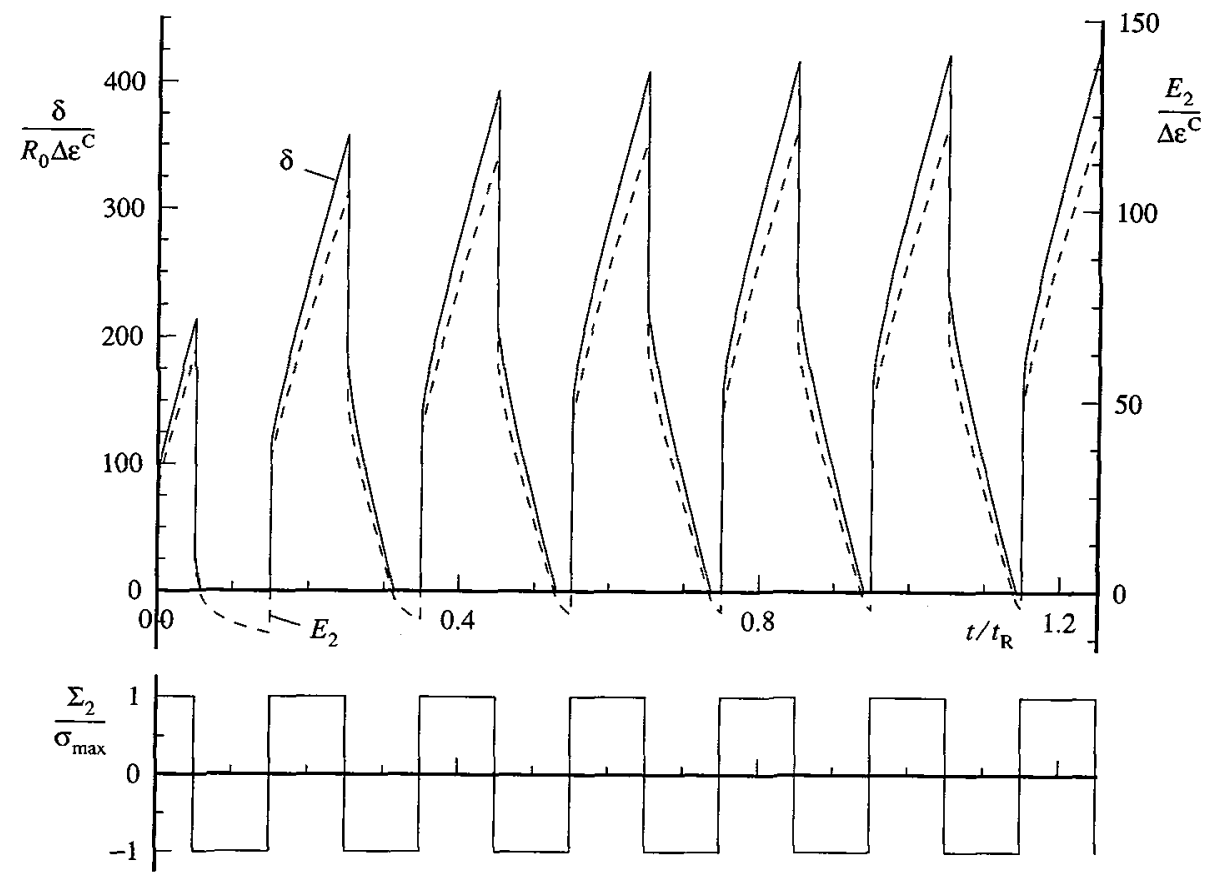

Figure 3: Evolution of microcrack opening $\delta$ in a microcracked material under balanced stress-controlled $\left(\Sigma_{2}\right)$ loading, along with the macroscopic strain response $E_{2}$. Due to the free grain boundary sliding, the microcrack opening is virtually uniform over the central facet.

in the next compression wave occurs only near the end of that period. During subsequent cycles, further settling of the residual stress field takes place, in such a way that after six or so cycles, a steady-state has been reached in which the crack faces just touch at the end of the compression waves. The fact that the macroscopic strain response is two orders of magnitude larger than the reference strain level $\Delta \epsilon^{\mathrm{C}}$ shows that microcrack opening and grain boundary sliding are the dominant contributions to $E_{2}$. Also notice that, at least during the tension periods, $\delta$ scales with $E_{2}$.

\subsection{Cyclic creep cavitation}

We proceed by considering now the case where the central facet is not cracked, but contains an initial, uniform distribution of small cavities with tip angle $\psi=75^{\circ}$. The initial spacing $b_{\mathrm{I}}$ of the cavities is taken as $0.1 R_{0}$, and the initial cavity size $a_{\mathrm{I}}$ is taken as small as $0.01 b_{\mathrm{I}}$ (it is recalled that at such a small size, the actual value of $a_{\mathrm{I}} / b_{\mathrm{I}}$ hardly affects the subsequent evolution [3]). The grain boundary diffusion parameter $\mathcal{D}$ in (3) is specified in terms of the ration between $a_{\mathrm{I}}$ and the value of $L$ according to (5) corresponding to the macroscopic effective stress $\Sigma_{e}$ and associated creep rate $\dot{E}_{\mathrm{e}}^{\mathrm{C}}$ (see the beginning of Sec. 3 ). The results in this section are for $(a / L)_{\mathrm{I}}=0.025$, which corresponds to conditions were cavity growth under creep conditions tends to be creep constrained (see $[3,7,4]$ ).

Figure 4 shows the damage evolution, in terms of $a / b$, along the central facet under the same balanced cyclic loading conditions as considered in Fig. 3. According to Fig. 4(a), cavity growth (and shrinkage) near the triple point is always faster than elsewhere, due to grain boundary sliding. 

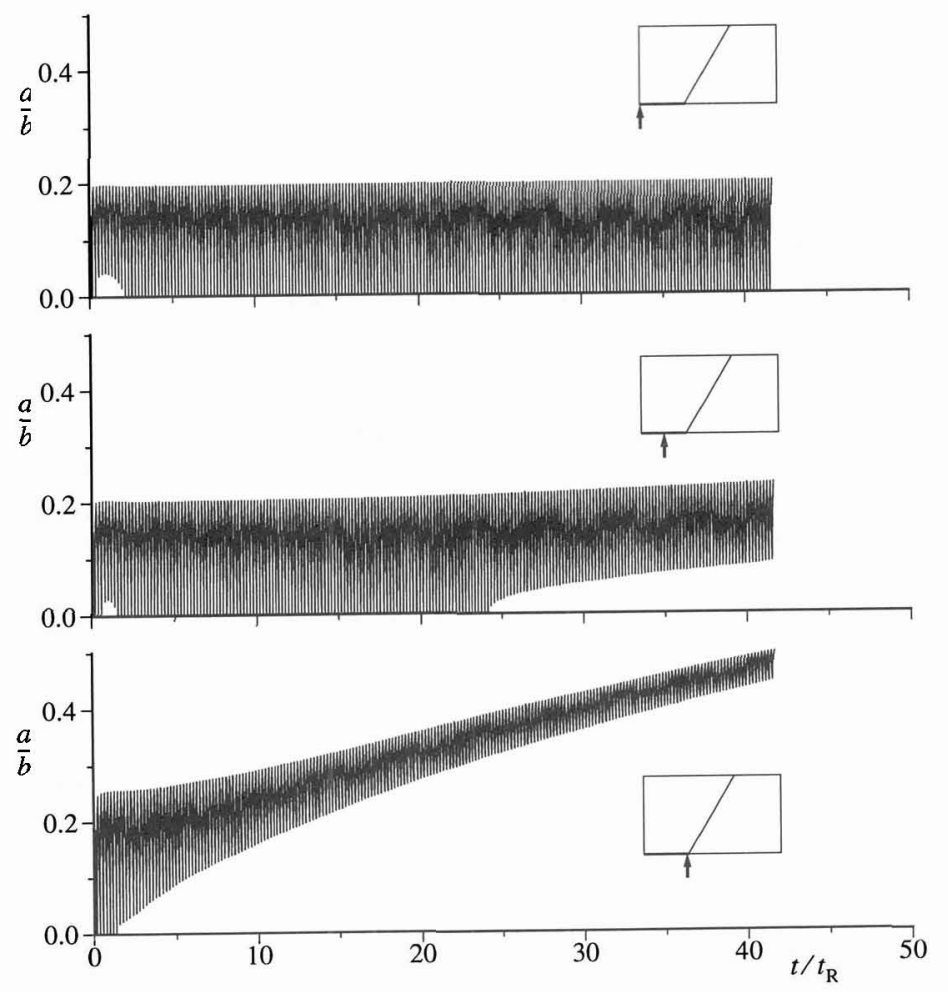

(a)

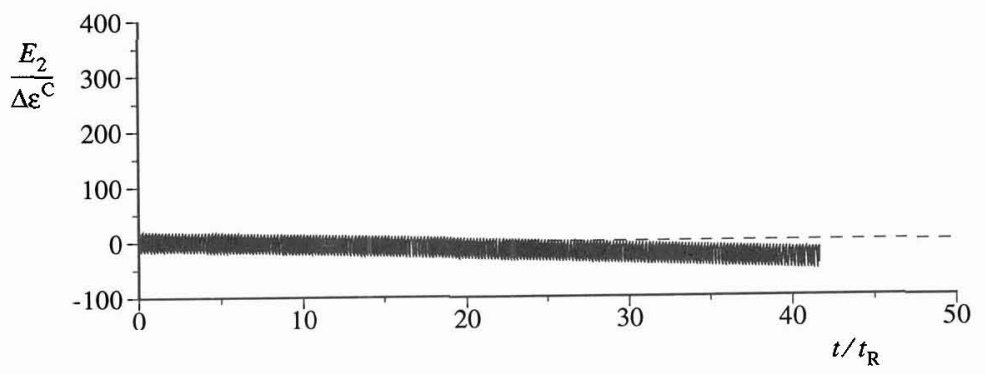

(b)

Figure 4: (a) Damage evolution during balanced cyclic loading $\left(t_{\mathbf{u}}=t_{1}, \sigma_{\min } / \sigma_{\max }=-1\right)$ at three points along the central facet. (b) Corresponding strain response showing a small amount of 'inverse ratchetting'.

But during the first few cycles, all cavities are completely closed down during the compression waves, while the peak damage levels then remain steady; this is consistent with the picture obtained in the previous section from the behaviour of an open micocrack (see also [8]). Next, there is a short time interval where residual stresses prevent cavities to completely close up, but the cycling between cavities opened up to about $a / b=0.2$ and just closed then gets restored over most of the facet, except near the triple point. There, presumably under the influence of sliding on the adjacent grain boundary, the cavities can no longer be fully closed, and the time-average of $a / b$ monotonically increases in time. As this takes place, the facet region where closure can not be maintained gradually spreads over the rest of the facet, as can clearly be seen from the results in Fig. 4 (a) near $x^{1} / R_{0} \approx 0.5$. Thus, it is expected that, even though the macroscopic loading is balanced, cavity coalescence will eventually take place near the triple point, with the resulting microcrack growing towards the center of the central facet. It is noted that a similar response 


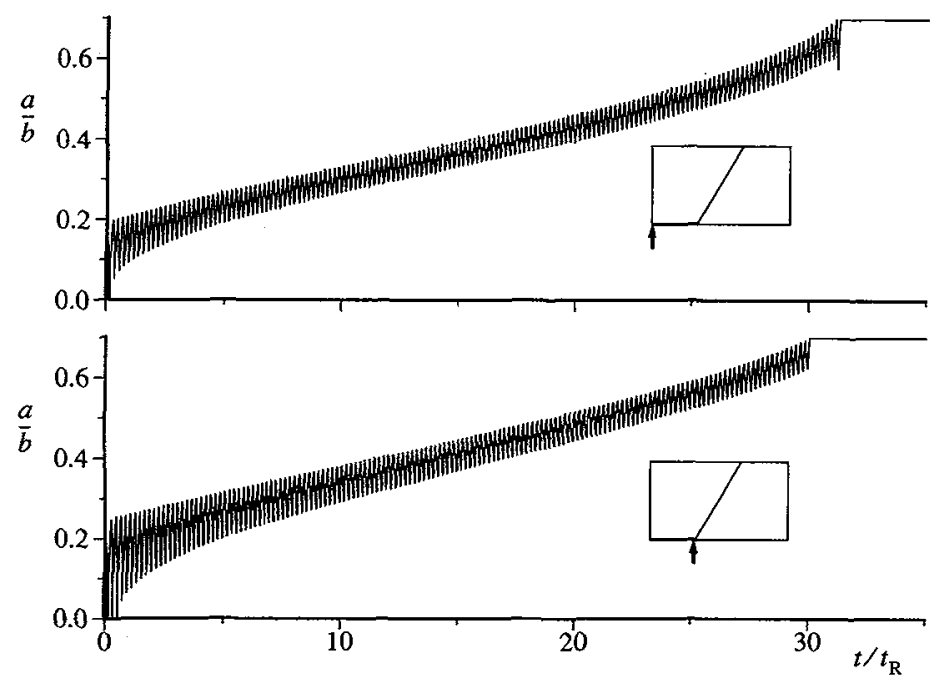

(a)

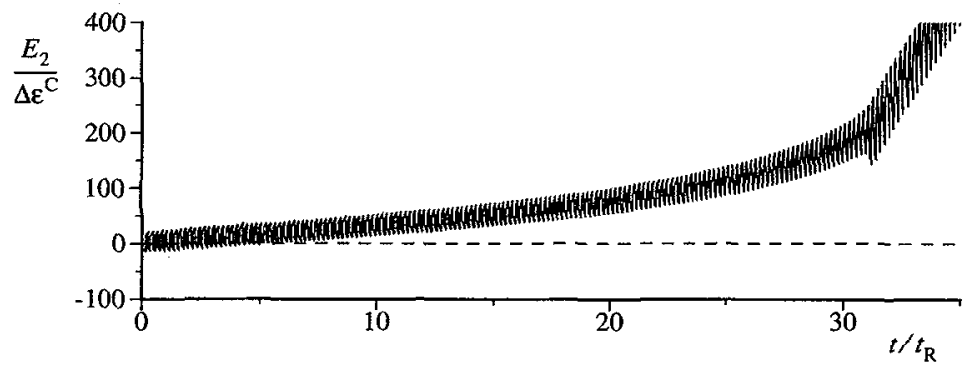

(b)

Figure 5: (a) Damage evolution at two points along the facet during unbalanced cyclic loading with $t_{\mathbf{u}}=t_{1}$, $\sigma_{\min } / \sigma_{\max }=-0.95$. (b) Corresponding ratchetting strain.

under strain-controlled cycling has been found in [8] for a corresponding value of $(a / L) \mathrm{r}$; but, for larger values of $(a / L)_{\mathrm{I}}$ (up to $(a / L)_{\mathrm{I}}=0.33$ ), where cavity growth is more creep controlled, the damage was found to oscillate steadily between 0 and some maximum value, without ever leading to coalescence.

The macroscopic strain behaviour is a result of the deformation and cavity growth processes taking place in the material in response to the prescribed, balanced stress-cycling. The resulting strain history in the $x^{2}$-direction of applied loading, shown in Fig. 4(b), exhibits a slow decrease of the time-average in time (for the purpose of comparison, this plot uses the same scale as in the forthcoming Fig. 5). At the same time, the time-average of the lateral strain $E_{1}$ (not shown) increases in time at roughly the same rate. This rather remarkable feature that the material tends to be gradually compressed and squeezed-out laterally, is to be attributed to the complex phenomenology of the spatially nonuniform damage evolution. As we have seen from Fig. 4(a), the area of the central facet where cavities get fully closed-up during a compression wave reduces in the course of the process. Hence, the prescribed compressive stress is transmitted through a shrinking region of the central facet, so that the 'contact stresses' and those in the undamaged grains during compression waves gradually grow, giving rise to a slow increase of the creep rate in compression. This then leads to the 'inverse ratchetting' effect shown in Fig. 4(b).

An example of the behaviour under a small amount of unbalance is shown in Fig. 5 for a stress cycle with $\sigma_{\min }=-0.95 \sigma_{\max }$ but otherwise identical conditions as in Fig. 4 . Now, there is a 


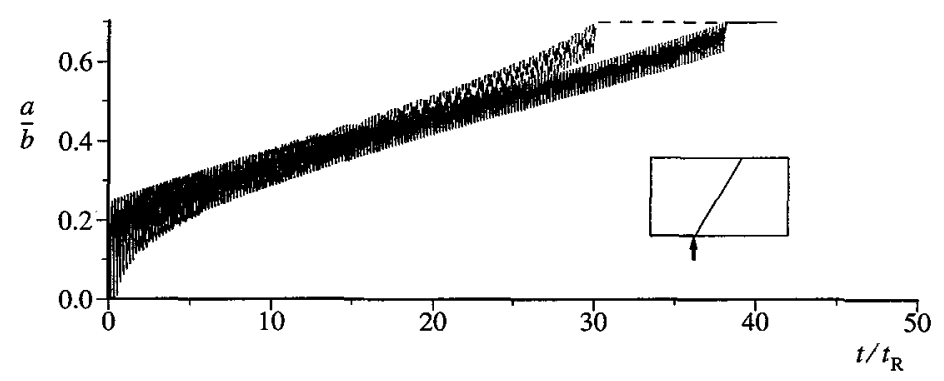

(a)

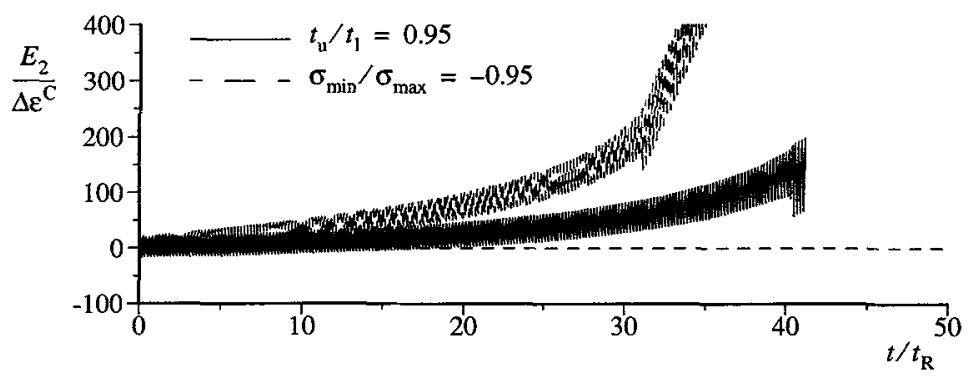

(b)

Figure 6: Comparison between cyclic loading with a time unbalance, $t_{\mathbf{u}}=0.95 t_{1}, \sigma_{\min } / \sigma_{\max }=-1$ (solid line), and the previous case of stress-unbalanced loading, $t_{\mathrm{v}}=t_{1}, \sigma_{\min } / \sigma_{\max }=-0.95$ (dashed line) shown in Fig. 5 . (a) Damage evolution near the triple point; (b) Corresponding ratchetting strain.

time-averaged net tensile stress, which is responsible for a time-average accumulation of damage, leading to cavity coalescence after about 150 cycles (see Fig. 5(a)). The actual value of $a / b$ oscillates about this time-average with an amplitude that is almost constant during most of the lifetime, but which is considerably smaller than that during balanced loading. It is also noted that the damage evolution is much more uniform over the facet than in the previous case of balanced loading. According to Fig. 5(b), this unbalanced cycling gives rise to substantial ratchetting as damage accumulates. Near the end of the lifetime, the ratchetting strain-rate increases somewhat; after the entire facet has failed, the ratchetting strain-rate as well as the amplitude of the oscillations accelerate as the compliance of the aggregate is increased. Notice that the jump in the ratchetting strain-rate upon facet failure indicates that the creep constraint on prior cavity growth has not been so strong as to reduce the normal stresses across the cavitating facets to negligible levels.

To demonstrate that cyclic creep depends in a subtle manner on the nonlinearities involved, Fig. 6 compares the above results with a case where $\sigma_{\min } / \sigma_{\max }=-1$ but where there is an unbalance in tension/compression hold times: $t_{\mathrm{u}}=0.95 t_{1}$. In both cases, the time average applied tensile stress, $\sigma_{\max } t_{1}+\sigma_{\min } t_{\mathrm{u}}$, is the same. If the cavity growth process would be governed by diffusion only, the cavity growth rates according to (2) and therefore the times to failure would be the same. With the chosen value of $(a / L)_{\mathrm{I}}=0.025$, diffusion is much faster than creep, but cavity growth in a polycrystalline aggregate tends to be constrained by creep. The resulting coupling between diffusion and creep is reflected in the fact that the stress and time unbalanced cases give rise to somewhat different damage accumulation processes (Fig. 6).

\section{DISCUSSION}

The investigation of intergranular creep failure under strain- controlled cyclic loading in [8] is here extended to also consider stress-controlled cyclic loading. As was found in [8], an analysis for an 
open facet-size microcrack gives important understanding of some features of cyclic creep damage behaviour. Thus, Fig. 3 for balanced cyclic stress loading shows that the initial shift between contact and loss of contact of the crack surfaces results in a redistribution of the stress and strain fields, so that contact only occurs briefly at the end of the compressive part of the cycle. This means that the average crack opening is positive, and the crack opening cycles between twice this average opening and zero opening. Based on these results, the expectation for a cavitating grain boundary facet in the creep constrained range is that under balanced cyclic loading the cavity volume will vary between zero and a more or less constant maximum value.

Some of the expected features are actually found in Fig. 4 for a cavitating facet in a material under balanced cyclic loading, with best agreement in the central part of the facet, while the behaviour near triple grain junctions differs rather quickly, due to a strong effect of grain boundary sliding. The cavities near the triple grain junctions grow much larger than expected based on the microcrack model, which may result in wedge crack formation even under balanced cyclic loading. In [8] a few of the computations without cavity nucleation were carried out for a small cavity density, $b_{\mathrm{I}} / R_{0}=1.0$, which makes the computations somewhat unrealistic; but it is noted that practically identical results are obtained here in Fig. 4 , for a realistic cavity density, $b_{\mathrm{I}} / R_{0}=0.1$, with the material subject to a relatively higher frequency of cycling.

A number of strain-controlled cases were presented in [8] where continuous nucleation of cavitities was accounted for during the tensile periods. When facets are under compression, no nucleation will take place, and this creates an unsymmetry in the response, even under balanced loading, which gives rise to a monotonic accumulation of the time-average damage. Qualitatively similar effects are to be expected under stress control.

Ratchetting is an important failure phenomenon, which has been considered here under stresscontrolled cycling. Such cyclic growth of the overall material straining can lead to a large accummulated strain, even though the addition in each cycle is small, and this can change the shape of a structural component enough to disqualify it for its purpose. Here, in Figs. 5 and 6 for unbalanced cyclic loading, the cyclic strain accummulation is studied together with the corresponding growth of intergranular creep damage. It is seen that in these cases grain boundary cavities grow rather rapidly, both at the centre of the grain boundary facet and near the triple grain junctions. Thus, unbalanced cycling, with a larger hold time in tension than in compression, or with a larger stress amplitude in tension, can lead to rather rapid formation of facet-size microcracks by cavity coalescence. The material may still have some remaining lifetime when such coalescence occurs, but it is seen in Figs. 5 and 6 that the rate of overall strain accummulation by ratchetting increases significantly after the formation of an open microcrack, which will tend to increase the rate of damage development in other parts of the material.

It should be noted that, certainly quantitatively, these results will depend on the assumed power-law creep model (1) for the grains. The phenomena discussed here, in particular the ratchetting effect, are a result of the interaction at the grain scale between diffusive grain boundary cavitation and creep deformations of the grain. Primary creep or typical cyclic plasticity phenomena may affect the observed behaviour; to assess the extent of such effects, more involved viscoplasticity models would have to be used instead of (1).

Also, completely free grain boundary sliding has been assumed here in order to maintain a relatively simple picture. For strain-controlled loading studied in [8], the opposite case of complete absence of grain boundary sliding has been explored as well. Even though, obviously, the profile of the opening facet crack (cf. Fig. 3) is different, the phenomenology of opening and closure of the crack was seen to be qualitatively similar to that with free sliding. This was also found for cyclic damage accumulation, with an expected reduction of the damage accumulation rate without sliding. There is no reason to expect that absence of sliding has a qualitatively different effect on the present results for stress-controlled cyclic behaviour. 


\section{REFERENCES}

[1] Cocks A.C.F., Ashby M.F., Progr. Mater. Sci. 27 (1982), 189-244.

[2] Needleman A., Rice J.R., Acta Metall. 28 (1980), 1315-1332.

[3] Tvergaard V., J. Mech. Phys. Solids 32 (1984), 373-393.

[4] Van der Giessen E., Tvergaard V., Acta Metall. Mater. 42 (1994), 959-973.

[5] Riedel H., Fracture at High Temperatures (Springer Verlag, Berlin, 1987).

[6] Pineau A., Mechanisms of creep-fatigue interactions, Advances in Fatigue Science and Technology, NATO ASI, Alvor April 1988, C. Moura Branco and L. Guerra Rosa Eds., (Kluwer Academic Publ., Dordrecht, 1989) pp. 283-311.

[7] Van der Giessen E., Tvergaard V., Mech. Mater. 17 (1994), 47-69.

[8] Van der Giessen E., Tvergaard V., Acta Mat. 44 (1996), 2697-2710.

[9] Budiansky B., Hutchinson J.W., Slutsky S., Mechanics of Solids, H.G. Hopkins and M.J. Sewell Eds., (Pergamon Press, Oxford, 1982) pp. 13-45.

[10] Sham T.-L., Needleman A., Acta Metall. 31 (1983), 919-926.

[11] Dyson B.F., Metal Science 10 (1976), 349-353. 